\title{
TUZA'S CONJECTURE FOR RANDOM GRAPHS
}

\author{
JEFF KAHN AND JINYOUNG PARK
}

\begin{abstract}
A celebrated conjecture of Zs. Tuza says that in any (finite) graph, the minimum size of a cover of triangles by edges is at most twice the maximum size of a set of edge-disjoint triangles. Resolving a recent question of Bennett, Dudek and Zerbib, we show that this is true for random graphs; more precisely:

for any $p=p(n), \mathbb{P}\left(G_{n, p}\right.$ satisfies Tuza's Conjecture $) \rightarrow 1$ (as $\left.n \rightarrow \infty\right)$.
\end{abstract}

\section{INTRODUCTION}

In this paper, we use matching and cover for triangle-matching and cover of triangles by edges, and $\nu$ and $\tau$ for the corresponding matching and cover numbers; thus, for a (finite) graph $H, \nu(H)$ is the maximum size of a set of edge-disjoint triangles and $\tau(H)$ is the minimum size of a set $F$ of edges with the property that each triangle contains a member of $F$.

We are interested in the celebrated Tuza's Conjecture:

Conjecture 1.1 (Tuza [16]). For any graph $H, \tau(H) \leq 2 \nu(H)$.

The inequality is tight when $H$ is $K_{4}$ or $K_{5}$ (or, e.g., a disjoint union of copies of these), and is not far from tight in other cases not related to these two examples (even if the graph is $K_{4}$-free; see [9]). We will not survey the literature—see e.g. [9,3]—and just mention that the best general result remains that of Haxell [8]:

$$
\text { for every } H, \tau(H) \leq \frac{66}{23} \nu(H) \text {. }
$$

Here we consider a question raised recently by Bennett, Dudek and Zerbib [3] and independently by Basit and Galvin [7]; informally: is Tuza's conjecture true for random graphs? More precisely, is it true that for any $p=p(n)$ and $G_{n, p}$ the usual binomial (or "Erdős-Rényi") random graph, w.h.p. ${ }^{1}$

$$
G_{n, p} \text { satisfies Tuza's Conjecture? }
$$

In [3] this was shown to be true if $p<c_{1} n^{-1 / 2}$ or $p>c_{2} n^{-1 / 2}$, with $c_{1} \approx 0.48$ and $c_{2} \approx 4.25$. (They work with $G_{n, m}$, but, as usual, this is about the same as $G_{n, p}$ with $p=m /\left(\begin{array}{l}n \\ 2\end{array}\right)$ and we will stick to the binomial version.) Here we finish this story:

Theorem 1.2. For any $p=p(n), \tau\left(G_{n, p}\right) \leq 2 \nu\left(G_{n, p}\right)$ w.h.p.

(This is in some sense a failure: for a while it seemed to us that the gap in [3] might hide counterexamples to Tuza's Conjecture.)

JK was supported by NSF Grants DMS1501962 and DMS1954035.

1“with high probability," meaning with probability tending to 1 as $n \rightarrow \infty$. 
We recently heard from Patrick Bennett that he, Ryan Cushman and Andrzej Dudek [2] have also closed the gap in [3], using an approach similar to that of the earlier paper (and different from what we do here).

For the rest of this paper we use $G$ for $G_{n, p}$, and set $m=\left(\begin{array}{l}n \\ 2\end{array}\right) p(=\mathbb{E}|G|)$ and $d=(n-2) p^{2}$ (the expected number of triangles on a given edge of $G$ ). Of course for the proof of Theorem 1.2 we could confine ourselves to $d$ in the range not covered by [3], but we will give arguments for the full range, in the process strengthening the earlier results.

To begin, for smallish $d$, we have an asymptotically optimal statement:

Theorem 1.3. If $d \leq 1 / 2$, then w.h.p. $\tau(G) \sim \nu(G)$.

(Of course $\tau(G) \geq \nu(G)$ is trivial.)

For treatment of larger $d=\Theta(1)$, set $\xi(d)=\frac{1}{3}\left[1-(2 d+1)^{-1 / 2}\right]$ and $\psi(d)=\frac{1}{2}\left[1-\exp \left(-\frac{d}{2}\left(1+e^{-d}\right)\right)\right]$. The next two assertions are our main points.

Theorem 1.4. If $d=\Theta(1)$, then w.h.p.

$$
\nu(G)>(1-o(1)) \xi(d) m
$$

Theorem 1.5. If $d=\Theta(1)$, then w.h.p.

$$
\tau(G)<(1+o(1)) \psi(d) m
$$

The proof of Theorem 1.2 for fixed $d \geq 1 / 2$ is then completed by the following calculation.

Lemma 1.6. For any $d \geq 1 / 2, \psi(d)<2 \xi(d)$.

A verification of the elementary (but not easy) Lemma 1.6 is sketched in Appendix A. Note the lemma is trivial for large enough $d$. It is actually true for all positive $d$, but its already annoying proof becomes even more annoying for $d$ below $1 / 2$ and, not needing this, we skip it. We provide (and possess) no insight suggesting that the lemma is more than a lucky coincidence. (It is sometimes just barely true; see Figure 1 in the Appendix.) On the other hand, we haven't much reason to think that $\nu$ isn't significantly larger than what we're able to show. (We guess Theorem 1.5, though slightly improvable, is close to the truth.)

Finally, completing the picture, we observe that for larger $d$, both $\nu$ and $\tau$ behave as one would expect. Here we recall that

$$
\text { for any } H, \nu(H) \leq|H| / 3 \text { and } \tau(H)<|H| / 2 \text {. }
$$

(The bound on $\nu$ is trivial and that on $\tau$ is the standard observation that on average, for a random equipartition $V(H)=X \cup Y$, more than half the edges of $H$ have ends in both $X$ and $Y$.) It turns out that as $d \rightarrow \infty$, both these bounds are (w.h.p.) asymptotically tight for $G$. For $\tau$ this is due to Frankl and Rödl [5] (see also [10, Theorem 8.14]; here, of course, it is just context, not part of the proof of Theorem 1.2). We will show:

Theorem 1.7. If $d \gg 1$ then w.h.p. $\nu(G) \sim m / 3$.

This is an easy consequence of Pippenger's Theorem (or a slight variant thereof; see Section 7), but despite some past interest (again, see Section 7), seems not to have been pointed out previously. 
Outline. Section 2 gives definitions (mostly involving "triangle-trees"), proves a few simple results concerning these, and recalls a little standard machinery. Section 3 introduces the breadth-first triangle-trees $S^{*}(x y)$ and establishes couplings-Corollary 3.3 in particular-that underlie Theorems 1.3-1.5. The latter are proved in Sections 4-6 respectively, and, as noted above, the proofs of Theorem 1.7 and Lemma 1.6 are given in Section 7 and Appendix A.

\section{BASICS}

2.1. Definitions. We use $H$ for a general graph, reserving $G$ for $G_{n, p}$ and $V$ for $V(G)(=[n])$. As is common, $H[X]$ is the subgraph of $H$ induced by $X, N(x)$ is the neighborhood of $x$ (in the graph under discussion), $N(x, y)=N(x) \cap N(y)$, and $\nabla(A, B)$ is the set of edges joining disjoint sets of vertices $A, B$. We tend to think of graphs as edge sets, and in particular write $|H|$ for $|E(H)|$.

We use $\mathcal{R}(H)$ for the set of triangles in $H$ and, for $\mathcal{A} \subseteq \mathcal{R}(H), V(\mathcal{A})$ and $E(\mathcal{A})$ for the sets of vertices and edges in triangles of $\mathcal{A}$.

A graph $T$ is a triangle-tree if it can be gotten by starting with an edge $\rho$ (the root of $T$ ) and repeatedly adding a triangle consisting of an already used edge and a not-previously-used vertex. A triangle-tree $T$ is a triangle-path if each new triangle uses an edge that was added in the preceding step.

We will sometimes use simply "tree" and "path" for "triangle-tree" and "triangle-path," since, with one tiny exception (in the proof of Proposition 2.3), these are the only trees and paths we will see.

The length of a (triangle-)path is its number of triangles. The distance, $\operatorname{dist}(a, b)$ between elements $a, b$ of $H$ (meaning members of $V(H) \cup E(H) \cup \mathcal{R}(H)$ ) is the length of a shortest path joining $a$ and $b$ (so $\operatorname{dist}(a, b)=\infty$ if there is no such a path, but we won't need this). The depth of an element of a tree is its distance to the root, and the depth of the tree itself is the largest of the depths of its elements.

For $A, B \in \mathcal{R}(T)$ ( $T$ a tree), we say $A$ is a child of $B$ (and $B$ is the parent of $A$ ) if $B$ is the triangle immediately preceding $A$ on the (unique) path from the root to $A$. Similarly, the base of an element $a$ of $T$ is the last edge preceding $a$ on the path joining the root to $a$ (so the edge that the first triangle containing $a$ shares with the triangle that preceded it).

We say $H$ is triangle-connected iff any two of its edges are connected by a triangle-path. In particular, a single edge is a triangle-path and triangle-connected. A triangle-component of $H$ is a maximal triangleconnected subgraph of $H$, and is trivial if it is a single edge. For $x y \in H$, we use $S(x y)$ for the trianglecomponent containing $x y$.

We may build any triangle-connected $S$ (say rooted at $e=x y$ ) by starting with $e$ and repeatedly adding triangles, each sharing at least an edge with what we already have. Thus the number of added vertices (i.e. other than $x, y$ ) is at most half the number of added edges, with

equality iff $S$ is a (triangle-)tree.

We use $S^{d}$ for the Galton-Watson-like (random, possibly infinite) triangle-tree gotten by starting with a root edge $\rho$ and letting each edge in turn give birth to a random number of triangles with distribution $\operatorname{Po}(d)$ (these choices made independently). This "ideal" tree is susceptible to exact analysis, and the proofs 
of Theorems 1.3-1.5 will involve comparing it with $S(x y)$. We will "interpolate" between them using a breadth-first triangle tree, $S^{*}(x y)$, to be defined in Section 3 .

Finally, we set $S_{\gamma}(x y)=\{e \in S(x y): \operatorname{dist}(x y, e) \leq \gamma\}$ and define $S_{\gamma}^{d}$ analogously (and similarly for $S_{\gamma}^{*}(x y)$ when we get there).

\subsection{Small Claims.}

Proposition 2.1. If $T$ is a finite triangle-tree, then $\tau(T)=\nu(T)$.

Proof. We proceed by induction on $|T|$. The statement is trivial if the depth, say $t$, of $T$ is 0 or 1 ; so assume $t \geq 2$ and let $A$ be a triangle of depth $t$. Let $e, f, g$ be the edges of $A$, with $e$ its base (so $A$ is the only triangle containing either of $f, g$ ); let $B$ be the parent of $A$; and let $e, h, k$ be the edges of $B$, with $h$ its base.

Let $\mathcal{U}$ be the set of triangles with base $e$ and notice that $T \backslash E(\mathcal{U})$ is the edge-disjoint union of two triangletrees, $T_{1}$ and $T_{2}$ (one of them, rooted at $k$, of depth at most 1). But then if $\mathcal{M}_{i}$ and $C_{i}$ are (resp.) a maximum matching and minimum cover of $T_{i}$, induction gives $\left|\mathcal{M}_{i}\right|=\left|C_{i}\right|$ for each $i$, so $\mathcal{M}:=\mathcal{M}_{1} \cup \mathcal{M}_{2} \cup\{A\}$ and $C:=C_{1} \cup C_{2} \cup\{e\}$ are a matching and cover of $T$ with $|\mathcal{M}|=|C|$, and the proposition follows.

In what follows we will be interested in trees that are not too deep, say of depth $\gamma=\gamma(n)$ satisfying

$$
\gamma \ll \log n / \log \log n .
$$

Proposition 2.2. For fixed $d, \gamma$ as in (3), and distinct $x, y \in V$, the probability that $x y \in G$ and $S_{\gamma}(x y)$ is not a tree is less than $n^{-1+o(1)}$.

Proof. We first observe that, assuming $x y \in G$, if $S:=S_{\gamma}(x y)$ is not a tree, then it contains, for some $i<2 \gamma$, a subgraph $T$ with $x y \in T,|V(T) \backslash\{x, y\}|=i$ and $|E(T) \backslash\{x y\}| \geq 2 i+1$. For if

$$
t=\min \left\{\alpha: S_{\alpha}(x y) \text { is not a triangle-tree }\right\}(\leq \gamma),
$$

then $S_{t}(x y)$ contains, for some vertex $v$, distinct triangle-paths $P_{1}$ and $P_{2}$ of length at most $t$ from $x y$ to $v$, and we have $i:=\left|V\left(P_{1} \cup P_{2}\right) \backslash\{x, y\}\right| \leq 2 t-1$ and $\left|E\left(P_{1} \cup P_{2}\right) \backslash\{x y\}\right| \geq 2 i+1$ (since $P_{1} \cup P_{2}$ is triangle-connected and not a tree; see (2)).

But the probability that $G$ contains such a $T$ is less than

$$
p \sum_{i \leq 2 \gamma-1}\left(\begin{array}{c}
n \\
i
\end{array}\right)\left(\begin{array}{c}
\left(\begin{array}{c}
i+2 \\
2
\end{array}\right) \\
2 i+1
\end{array}\right) p^{2 i+1}=(O(\gamma))^{\gamma} n^{-1}
$$

(with the initial $p$ for $x y \in G$ ), which is $n^{-1+o(1)}$ for $\gamma$ as in (3).

Proposition 2.3. For the random triangle-tree $S^{d}$ :

(a) $S^{d}$ is finite with probability 1 iff $d \leq 1 / 2$;

(b) the expected number of triangles of depth $i$ in $S^{d}$ is $(2 d)^{i} / 2$.

Proof. These are basic properties of a Galton-Watson (GW) process (e.g. [12, Section 5.1]). We may associate with $S^{d}$ the (ordinary) random tree $U$ with $V(U)=E\left(S^{d}\right)$ and $f$ a child of $e$ in $U$ iff, in $S^{d}, e$ is the base of $f$. Then $U$ is a GW tree with the number of children of each $e$ distributed as $L=2 \mathrm{Po}(d)$. The assertions (a) and (b) are then given by Propositions 5.4 and 5.5 of [12], which say (resp.) that $U$ is finite with probability 1 iff $\mathbb{E} L \leq 1$ (unless $L \equiv 1$ ), and that the expected number of vertices at depth $i$ in $U$ is $(2 d)^{i}$. 
2.3. Concentration. We need two standard concentration facts (for the first see e.g. [10, Theorem 2.1]).

Theorem 2.4. If $\xi$ is binomial with $\mathbb{E} \xi=\mu$, then for $t \geq 0$,

$$
\begin{aligned}
& \operatorname{Pr}(\xi \geq \mu+t) \leq \exp \left[-t^{2} /(2(\mu+t / 3))\right], \\
& \operatorname{Pr}(\xi \leq \mu-t) \leq \exp \left[-t^{2} /(2 \mu)\right] .
\end{aligned}
$$

The second fact is "McDiarmid's Inequality." (It is also called, for example, the Hoeffding-Azuma Inequality. It is not the best one can say in the situations below, but is enough for our purposes.)

Theorem 2.5 ([13], Lemma 1.2). Let $X_{1}, \ldots, X_{l}$ be independent random variables, with $X_{k} \in A_{k}$ for each $k$. Suppose the (measurable) function $f: \prod A_{k} \rightarrow \mathbb{R}$ satisfies, for each $k$,

$$
\left|f(X)-f\left(X^{\prime}\right)\right| \leq c_{k}
$$

whenever $X=\left(X_{i}: i \in[l]\right)$ and $X^{\prime}=\left(X_{i}^{\prime}: i \in[l]\right)$ differ only in their $k$ th coordinates.

Then for any $t>0$,

$$
\mathbb{P}(|f-\mathbb{E} f| \geq t) \leq 2 \exp \left[-2 t^{2} / \sum c_{k}^{2}\right]
$$

We will always use this with $l=\left(\begin{array}{c}n \\ 2\end{array}\right)$ and $X_{i}=\mathbf{1}_{\left\{e_{i} \in G\right\}}$, where $E\left(K_{n}\right)=\left\{e_{i}: i \in[l]\right\}$ (so $X=G$ ), in which case we have

$$
\text { if } f \text { is Lipschitz (i.e. satisfies (4) with } c_{k}=1 \forall k \text { ) and } \mathbb{E} f \gg n \text {, then } f \sim \mathbb{E} f \text { w.h.p. }
$$

2.4. Number of subgraphs. For a graph $H$, let $\rho(H)=|E(H)| /|V(H)|$ (the density of $H$ ), and say $H$ is balanced if every $H^{\prime} \subseteq H$ has $\rho\left(H^{\prime}\right) \leq \rho(H)$.

Theorem 2.6 ([1], Theorem 4.4.4). Let $H$ be balanced with $v$ vertices, e edges and a automorphisms, and let $X$ be the number of copies of $H$ in $G_{n, p}$. If $p \gg n^{-v / e}$ then w.h.p.

$$
X \sim n^{v} p^{e} / a
$$

2.5. Binomial v. Poisson. For our limited purposes we use simply $\|X-Y\|$ for the total variation distance between discrete random variables $X$ and $Y$; this is (by definition) half the $l_{1}$ distance between their distributions, and is the minimum of $\mathbb{P}(X \neq Y)$ under couplings of $X$ and $Y$.

Proposition 2.7. For $n$ and $c \geq-n$ integers, $p \in[0,1], X \sim \operatorname{Bin}(n+c, p)$ and $Y \sim \operatorname{Po}(n p)$,

$$
\|X-Y\| \leq|c| p+O(p) .
$$

Proof. Let $Z \sim \operatorname{Bin}(n, p)$. Since $\|X-Y\| \leq\|X-Z\|+\|Z-Y\|$, and $\|Z-Y\|=O(p)$ (see (1.5) of [17] for a precise statement), it is enough to show $\|X-Z\| \leq|c| p$. To see this, we may couple $X$ and $Z$ by setting $l=\max \{n, n+c\}$, letting $\xi_{1} \ldots \xi_{l}$ be independent with $\xi_{i} \sim \operatorname{Ber}(p)(\forall i)$, and setting $Z=\sum_{i \leq n} \xi_{i}$ and $X=\sum_{i \leq n+c} \xi_{i}$, yielding $\mathbb{P}(X \neq Z) \leq|c| p$. 


\section{BREADTH FIRST AND BRANCHING}

We use $d(x)$ for the degree of $x$ (in $G$ ) and $x y z$ for the triangle with vertices $x, y, z$, and assume in this section that $d\left(=(n-2) p^{2}\right)=\Theta(1)$.

As mentioned earlier, the proofs of Theorems 1.3-1.5 depend on linking $S(x y)$ with the ideal triangle tree $S^{d}$ defined at the end of Section 2.1, a connection based on comparing each of these with the breadth-first triangle-tree rooted at $x y \in G$. This is the $x y$-rooted triangle-tree gotten by processing edges in the order in which they enter the tree, where processing $u v$ means adding all triangles $u v w$ with $w$ a vertex not yet in the tree; more formally:

Fix an order $\prec$ on $V$, set $P_{0}=\{x, y\}$, and let $\mathcal{R}_{0}$ be the set of triangles on $x y, V_{0}=V\left(\mathcal{R}_{0}\right)$ and $Q_{0}=$ $V_{0} \backslash P_{0}(=N(x, y))$. We process vertices $v_{1}, \ldots$ (this processing defined below), producing a sequence $\left(\mathcal{R}_{i}, P_{i}, Q_{i}, X_{i}\right)$. Each $\mathcal{R}_{i}$ will be the set of triangles of a tree, with $V_{i}:=V\left(\mathcal{R}_{i}\right)=P_{i} \amalg Q_{i}$ and $X_{i}:=V \backslash V_{i}$. When we finish processing $v_{i}$, vertices of $P_{i}$ have been processed and vertices of $Q_{i}$ are "in the queue" (in the tree and waiting to be processed). Of course we stop when the queue is empty, producing $S^{*}(x y)$.

We process vertices in the order in which they enter the evolving $V_{i}$, breaking ties according to $\prec$. A key property that will hold throughout the evolution (which basically says we are building a tree) is

$$
\text { each } v \in Q_{i} \text { lies in a unique triangle, } T \text {, of } \mathcal{R}_{i} \text {, and } V(T) \backslash\{v\} \subseteq P_{i} \text {. }
$$

Processing $v=v_{i}\left(\in Q_{i-1}\right)$ means: with $a b$ the base of $v$ we form $\mathcal{R}_{i}$ by adding to $\mathcal{R}_{i-1}$ all triangles avw and bvw with

$$
w \in X_{i-1} .
$$

So we may - this will be natural below-also think of this as processing the edges av and $b v$, and each $w$ as above enters $Q_{i}$ with base one of $a v, b v$. Note there is no ambiguity here:

$$
N(a, b) \cap X_{i-1}=\emptyset,
$$

since any $u \in N(a, b)$ not already in the tree when $a b$ was processed would have been added to the tree at that time. We then (in addition to $\mathcal{R}_{i}$ ) update $P_{i}=P_{i-1} \cup\left\{v_{i}\right\}$ and define $V_{i}, Q_{i}, X_{i}$ as above (so $Q_{i}$ is $Q_{i-1} \backslash\left\{v_{i}\right\}$ plus the $w^{\prime}$ s added at (7)).

Notice that this supports (6), which is true when $w$ enters the tree, and remains so until $w$ is processed and removed from $Q_{i}$ (since none of the intervening steps involves edges at $w$ ).

Proposition 3.1. For any $\gamma$, if $S_{\gamma}(x y)$ is a triangle-tree, then $S_{\gamma}^{*}(x y)=S_{\gamma}(x y)$.

Proof. Set $S=S(x y)$ and $S^{*}=S^{*}(x y)$. Notice to begin that if $e, f$ are edges of $S^{*}$ with $e$ processed before $f$, then, with dist* denoting distance in $S^{*}$, $\operatorname{dist}^{*}(x y, e) \leq \operatorname{dist}^{*}(x y, f)$ (by induction: the base, $e^{\prime}$, of $e$ was processed no later than the base, $f^{\prime}$, of $f$, so $\left.\operatorname{dist}^{*}(x y, e)=\operatorname{dist}^{*}\left(x y, e^{\prime}\right)+1 \leq \operatorname{dist}^{*}\left(x y, f^{\prime}\right)+1=\operatorname{dist}^{*}(x y, f)\right)$.

Suppose the proposition fails and let $A=u v w \in \mathcal{R}\left(S_{\gamma}\right) \backslash \mathcal{R}\left(S_{\gamma}^{*}\right)$ with $\operatorname{dist}(x y, A)$ minimum and $e=u v$ the base of $A$ in the unique path $P$ from $x y$ to $A$ in $S_{\gamma}$. Then $\mathcal{R}(P) \backslash\{A\} \subseteq \mathcal{R}\left(S_{\gamma}^{*}\right)$ implies $e$ was processed in the construction of $S^{*}$ and $A$ was not added, so $w$ was added before $e$ was processed. But then, by the observation in the last paragraph, $\operatorname{dist}^{*}(x y, w) \leq \operatorname{dist}^{*}(x y, e)+1 \leq \gamma$. Thus $S_{\gamma}$ contains two distinct paths from $x y$ to $w$, contradicting the assumption that $S_{\gamma}$ is a tree. 
For Proposition 3.2 and Corollary 3.3 we assume $\gamma$ is as in (3).

Proposition 3.2. On $\{x y \in G\}$, we may couple $S_{\gamma}^{*}(x y)$ and $S_{\gamma}^{d}$ so that they are equal w.h.p.

Combining this with Propositions 2.2 and 3.1 gives our main point:

Corollary 3.3. On $\{x y \in G\}$, we may couple $S_{\gamma}(x y)$ and $S_{\gamma}^{d}$ so that they are equal w.h.p.

Proof of Proposition 3.2. We think of generating $S^{*}:=S^{*}(x y) \subseteq G$ by exposing edges as needed, where "exposing" an edge is deciding whether it's in $G$. Precisely: we expose $\nabla(\{x, y\}, V \backslash\{x, y\})$, thus specifying the triangles of $S^{*}$ containing $x y$, and then, for $i=1, \ldots, \nabla\left(v_{i}, X_{i-1}\right)$, determining the triangles added in the processing of $v_{i}$.

The number of triangles on $x y$ has law $\operatorname{Bin}\left(n-2, p^{2}\right)$. When we process $v:=v_{i}$, say with base $a b$, the number of triangles added on $a v$ (and similarly for $b v$ ) has law $\operatorname{Bin}\left(\left|N(a) \cap X_{i-1}\right|, p\right)$ (note we do know $N(a) \cap X_{i-1}$ at this point), which will usually be close to $\operatorname{Po}(d)$, since $\left|N(a) \cap X_{i-1}\right|$ is usually close to $n p$.

We may think of a parallel generation of $S^{d}$ : when processing an edge $e$ in the generation of $S^{*}$, we simultaneously specify the number of triangles on $e$ in $S^{d}$, coupling so that the numbers of triangles in these two choices agree as often as possible. Once the numbers agree, we may couple so the trees themselves do as well. Of course this only makes sense as long as the trees agree: if and when they do not, the coupling has failed and we lose interest.

It remains to bound the probability that the coupling fails. Set (with plenty of room) $\kappa=n^{0.1}$ and $\varsigma=n^{-0.2}$, and define events $Q_{1}=\left\{\left|S_{\gamma}^{d}\right|>\kappa\right\}$ and $Q_{2}=\{\exists v \in V d(v) \neq(1 \pm \varsigma) n p\}$. Proposition 2.3(b) (with Markov's Inequality) and Theorem 2.4 imply $\mathbb{P}\left(Q_{1}\right)=o(1)$ and $\mathbb{P}\left(Q_{2}\right)=n \exp \left[-\Omega\left(\varsigma^{2} n p\right)\right](=o(1))$.

For the coupling, we use Proposition 2.7, noting to begin that it bounds the probability of failure when we process $x y$ by $\left\|\operatorname{Bin}\left(n-2, p^{2}\right), \operatorname{Po}(d)\right\|=O\left(p^{2}\right)$.

Suppose we have successfully coupled through the processing of $v_{i-1}$ and let $a b$ be the base of $v_{i}$. The probability that the coupling now fails at (e.g.) $a v_{i}$ is at most

$$
O\left(p\left[|| N(a) \cap X_{i-1}|-(n-2) p|+1\right]\right),
$$

which is $O((\varsigma n p+\kappa) p)=O\left(n^{-0.2}\right)$ provided $d(a)=(1 \pm \varsigma) n p$ and $\left|V_{i-1}\right|<\kappa$. Thus the (overall) probability that the coupling fails is at most

$$
\mathbb{P}\left(Q_{1}\right)+\mathbb{P}\left(Q_{2}\right)+O\left(p^{2}+\kappa n^{-0.2}\right)=o(1) .
$$

\section{Proof Of THEOREM 1.3}

Assume first that $d=\Omega(1)$. Since $d \leq 1 / 2$, Proposition 2.3(a) implies that on $\{x y \in G\}$, under the coupling of Corollary 3.3, $S(x y)=S^{d}$ w.h.p. (namely, $S(x y)=S^{d}$ if $S_{\gamma}(x y)=S_{\gamma}^{d}$ and the depth of $S^{d}$ is less than $\gamma$, each of which is true w.h.p.). Since $S^{d}$ is a tree, this implies that the expected number of edges (of $G)$ in triangle components that are not trees is $o(m)$, so the actual number is $o(m)$ w.h.p.

Now let $G_{i}$ run over the triangle components of $G$ and notice that, trivially,

$$
\nu(G)=\sum \nu\left(G_{i}\right)
$$


and similarly for $\tau$. So, letting $\sum^{\prime}$ denote sum only over $G_{i}$ 's that are trees, and recalling Proposition 2.1, we have (w.h.p.)

$$
\tau(G)=\sum^{\prime} \tau\left(G_{i}\right)+o(m)=\sum^{\prime} \nu\left(G_{i}\right)+o(m)=\nu(G)+o(m) .
$$

This gives Theorem 1.3 when combined with

$$
\nu(G)=\Omega(m) \text { w.h.p. }
$$

Proof of (8). By (5) it's enough to show $\mathbb{E} \nu(G)=\Omega(m)$. But $\nu(G)$ is at least the number of isolated triangles in $G$ (an isolated triangle being one sharing no edges with other triangles), and the expected number of these is exactly

$$
\left(\begin{array}{l}
n \\
3
\end{array}\right) p^{3}\left(1-3 p^{2}+2 p^{3}\right)^{n-3}=\Omega(m) .
$$

(Of course this—with the asymptotics of the number of isolated triangles-could also be read off from the coupling with $S^{d}$.)

Now suppose $d \ll 1$ (i.e. $p \ll n^{-1 / 2}$ ). Let $Y$ be the number of triangles in $G, Y^{\prime}$ the number of nonisolated triangles and $X$ the number of edges that lie in exactly one triangle. For Theorem 1.3 it is enough to show that w.h.p. $Y^{\prime} \ll Y$ (i.e. almost all triangles are isolated).

For $p \ll n^{-4 / 5}$ we just observe that $Y^{\prime}=0$ w.h.p., since the expected number of pairs of triangles sharing an edge is $O\left(n^{4} p^{5}\right)$. For larger $p\left(p \gg 1 / n\right.$ is enough here), notice that $X \leq 3 Y-Y^{\prime}$. From Theorem 2.6 we have

$$
\text { w.h.p. } Y \sim \mathbb{E} Y=\left(\begin{array}{l}
n \\
3
\end{array}\right) p^{3} \sim n^{3} p^{3} / 6 \text {, }
$$

while $p \ll n^{-1 / 2}$ gives $\mathbb{E} X=\left(\begin{array}{l}n \\ 2\end{array}\right) p(n-2) p^{2}\left(1-p^{2}\right)^{n-3} \sim n^{3} p^{3} / 2 \sim 3 \mathbb{E} Y$. But then $\mathbb{E} Y^{\prime} \leq 3 \mathbb{E} Y-\mathbb{E} X \ll \mathbb{E} Y$ implies $Y^{\prime} \ll \mathbb{E} Y$ w.h.p., which with (9) gives $Y^{\prime} \ll Y$ w.h.p.

\section{Proof of Theorem 1.4}

Given a graph $H$ and $w: \mathcal{R}(H) \rightarrow[0,1]$ (values of $w$ will always be called weights), we use $\mathcal{M}_{w}^{*}$ for the greedy (triangle-)matching corresponding to $w$; namely: we consider triangles in (increasing) order of their weights, and at each step add the triangle under consideration to $\mathcal{M}_{w}^{*}$ iff it shares no edge with any triangle already in the matching. In particular when

$$
w \text { is uniform from }[0,1]^{\mathcal{R}(H)},
$$

$\mathcal{M}^{*}:=\mathcal{M}_{w}^{*}$ is the usual random greedy matching of $H$. (Strictly speaking we have defined $\mathcal{M}_{w}^{*}$ only when the weights are distinct; but for $w$ as in (10), this is true with probability 1 and will not be a concern.)

We will show that for any $x, y \in V$,

$$
\mathbb{P}\left(x y \notin E\left(\mathcal{M}^{*}\right) \mid x y \in G\right) \rightarrow(2 d+1)^{-1 / 2},
$$

where $\mathbb{P}$ refers to the choices of $G$ and $w$. This implies $\mathbb{E} \nu(G)>(1-o(1))(2 d+1)^{-1 / 2} m$, which with (5) gives Theorem 1.4. The proof of (11), which is inspired by [15], is based on the connection with $S^{d}$ in Corollary 3.3. We need a few simple notions and observations. 
For a finite triangle-tree $T$ we work with the following recursive survival rule for edges, in which we may evaluate edges in any order for which each edge appears earlier than its base (further specification of the order doesn't affect the outcome), and "dies" means fails to survive:

$$
e \text { dies iff it is the base of a triangle whose other two edges survive. }
$$

(For example, any edge that is the base of no triangle survives.)

For a general graph $H, e \in H$ and $w$ as in (10), let

$T(e)=\{f \in H:$ there is a triangle-path from $e$ to $f$ on which the weights of the triangles decrease $\}$

(a random subgraph of $S(e)$ ).

It is easy to see that if $T(e)$ is a tree then $e$ is covered by $\mathcal{M}^{*}$ iff it dies when we apply (12) to $T(e)$. (In this case it's natural to think of evaluating edges in increasing order of their weights. The present survival rule is the same as that of [15] applied to the (3-uniform) hypertree with vertices $E(T)$ and edges $\mathcal{R}(T)$ (and the natural incidences).)

When $H=S^{d}$ we use $T^{d}$ for $T(\rho)$ (recall $\rho$ is the root of $S^{d}$ ). As for (11), when we speak of $T^{d}$ (in Proposition 5.1 and Lemma 5.2), "probability" refers to the choices of both $S^{d}$ and $w$.

Proposition 5.1. $T^{d}$ is finite with probability 1.

Proof. By Proposition 2.3(b), the expected number of triangles of depth $i$ in $T^{d}$ is $(2 d)^{i} /(2 i !)$, which tends to zero as $i \rightarrow \infty$.

Proposition 5.1 and Corollary 3.3 imply

$$
\text { on }\{x y \in G\} \text { we may couple } T(x y) \text { and } T^{d} \text { to agree w.h.p. }
$$

(namely, we can couple so $T(x y)=T^{d}$ whenever $S_{\gamma}(x y)=S_{\gamma}^{d}$ and the depth of $T^{d}$ is less than $\gamma$ ).

In view of the preceding comments, this says that the probability in (11) tends to the probability that the root survives in $T^{d}$; so the proof of (11) (and Theorem 1.4) is completed by the following calculation.

Lemma 5.2. Under (12) the root of $T^{d}$ survives with probability $(2 d+1)^{-1 / 2}$.

Proof. It will be convenient to extend $w$ to edges: set $w(\rho)=1$, and for any other $e \in E\left(T^{d}\right)$ let $w(e)$ be the weight of the (unique) triangle on $e$ with minimum depth.

Let $f(x)$ be the probability that an edge of weight $x$ survives. Trivially, $f(0)=1$. The survival rule (12) says that an edge $e$ dies iff there is a child (triangle) of $e$ in $S^{d}$, say with edges $e, j, k$, such that

$$
(w(j)=) w(k)<w(e) \text { and both } j \text { and } k \text { survive. }
$$

Given $w(e)$ (with $e, j, k \in S^{d}$ as above), the probability of (13) is $\int_{0}^{w(e)} f^{2}(y) d y$, implying

$$
f(x)=\sum_{k} \mathbb{P}(Z=k)\left[1-\int_{0}^{x} f^{2}(y) d y\right]^{k}
$$


where $Z \sim \operatorname{Po}(d)$. Rewriting (14) with $F(x)=\int_{0}^{x} f^{2}(y) d y$ gives

$$
F^{\prime}(x)=\left[\sum_{k} \mathbb{P}(Z=k)(1-F(x))^{k}\right]^{2}=e^{-2 d F(x)} ; \quad F(0)=0 .
$$

The solution to this is

$$
F(x)=\frac{1}{2 d} \ln (2 d x+1)
$$

so we have

$$
f(x)=F^{\prime}(x)^{1 / 2}=(2 d x+1)^{-1 / 2},
$$

and the lemma follows.

\section{Proof of TheOrem 1.5}

For a partition $X \cup Y$ of $V=V(G)$ (we call each of $X, Y$ a block), let $W=W(X, Y)=\left(W_{0} \backslash W_{1}\right) \cup W_{2}$, where:

- $W_{0}=G[X] \cup G[Y]$

- $W_{1}=\left\{x y \in W_{0}\right.$ : all triangles on $x y$ are contained in the same block as $\left.x y\right\}$;

- $W_{2}=\left\{x y \in W_{1}\right.$ : there is a triangle $x y z$ with $\left.x z, y z \in W_{1}\right\}$.

It is easy to see that $W$ is a cover of $G$.

For Theorem 1.5, again by (5), it suffices to show that for $X \cup Y$ a uniformly random partition of $V$ (so each $v \in V$ is in $X$ with probability $1 / 2$, these choices made independently) and $W=W(X, Y)$,

$$
\mathbb{P}(x y \in W \mid x y \in G) \rightarrow \frac{1}{2}\left[1-\exp \left(-\frac{d}{2}\left(1+e^{-d}\right)\right)\right] .
$$

Proof of (15). Set $Q=\{x y \in G\}$ and note to begin that

$$
\mathbb{P}\left(x y \in W_{0} \mid Q\right)=1 / 2 .
$$

Set $p_{k}=e^{-d} d^{k} / k$ !. On $Q$ the distribution of the number of triangles on $x y$ is $\operatorname{Bin}\left(n-2, p^{2}\right) \stackrel{\mathrm{d}}{\longrightarrow} \operatorname{Po}(d)$, so

$$
\mathbb{P}\left(x y \in W_{1} \mid Q\right) \sim \sum_{k \geq 0} p_{k} 2^{-k-1}=e^{-d / 2} / 2 .
$$

For $W_{2}$ we use Corollary 3.3, now with $\gamma=2$. Assigning vertices of $S_{2}^{d}$ to $X$ and $Y$ in the same way as vertices of $V$ (i.e. via independent fair coin tosses), we may extend the coupling of the corollary to these choices so that $x y \in W_{2} \Leftrightarrow \rho \in W_{2}$ whenever $S_{2}(x y)=S_{2}^{d}$; yielding

$$
\mathbb{P}\left(x y \in W_{2} \mid Q\right)=\mathbb{P}\left(\rho \in W_{2}\right)+o(1)
$$

(where as usual $o(1)$ can be negative). On the other hand,

$$
\mathbb{P}\left(\rho \in W_{2}\right)=\sum_{k \geq 1} p_{k} 2^{-k-1}\left(1-(1-\alpha)^{k}\right),
$$

where

$$
\alpha=\sum_{l, m \geq 0} p_{l} p_{m} 2^{-l} 2^{-m}=\left[e^{-d} \sum_{l \geq 0} \frac{d^{l} 2^{-l}}{l !}\right]^{2}=e^{-d}
$$


is the probability that, given $\{x, y, z\} \subseteq X$ (e.g.), all triangles containing either of $x z, y z$ also lie in $X$. Then rewriting the r.h.s. of (19) as

$$
\frac{e^{-d}}{2}\left[e^{d / 2}-\exp \left(\frac{d}{2}\left(1-e^{-d}\right)\right)\right]
$$

and combining with (16)-(18) gives (15).

\section{Proof of THeOReM 1.7}

For $d>\log ^{3+\epsilon} n$ Theorem 1.7 was proved (somewhat implicitly) in [4], and, as observed in [3], direct application of Pippenger's Theorem improves this to $d \gg \log n$, where w.h.p. each edge of $G$ is in $(1+$ $o(1)) d$ triangles. (Pippenger's Theorem was never published and first appeared in [6]; see also e.g. [1, Theorem 4.7.1].)

In fact Pippenger's Theorem can also be used to prove Theorem 1.7, but we will find it convenient to use the following variant, a simplest instance of [11, Theorem 1.5]. (For fractional things see e.g. [14].)

For a hypergraph $\mathcal{H}$ and $\varphi: \mathcal{H} \rightarrow[0,1]$, let

$$
\alpha(\varphi)=\max \sum\{\varphi(A): x, y \in A \in \mathcal{H}\}
$$

the max over distinct vertices $x, y$ of $\mathcal{H}$.

Theorem 7.1. For fixed $r$, if $\mathcal{H}$ is $r$-uniform and $\varphi: \mathcal{H} \rightarrow[0,1]$ is a fractional matching, then

$$
\nu(\mathcal{H})>(1-o(1)) \sum_{A \in \mathcal{H}} \varphi(A),
$$

where o(1) $\rightarrow 0$ as $\alpha(\varphi) \rightarrow 0$.

(The statement in [11] also assumes $\sum \varphi(A) \rightarrow \infty$, but this is easily seen to be unnecessary.)

We will (of course) use Theorem 7.1 with $\mathcal{H}=\mathcal{R}(G)$ (and $V(\mathcal{H})=E(G)$ ). Let $1 \gg \varsigma \gg d^{-1 / 2}$ (recall $d \gg 1)$ and $D=(1+\varsigma) d$. Say $e \in E(G)$ is heavy if it lies in at least $D$ triangles, and define the fractional matching $\varphi: \mathcal{H} \rightarrow[0,1]$ by

$$
\varphi(A)= \begin{cases}1 / D & \text { if } A \text { contains no heavy edges, } \\ 0 & \text { otherwise. }\end{cases}
$$

Of course $\alpha(\varphi) \rightarrow 0$, so to get Theorem 1.7 from Theorem 7.1 we just need

$$
\text { w.h.p. } \sum \varphi(A) \sim m / 3 \text {; }
$$

this will follow from

$$
\text { w.h.p. the number of triangles of } G \text { containing heavy edges is } o\left(n^{3} p^{3}\right) \text {. }
$$

For if (20) is true then, since $|\mathcal{R}(G)| \sim\left(\begin{array}{l}n \\ 3\end{array}\right) p^{3}$ w.h.p. (see Theorem 2.6) and $D \sim n p^{2}$, we have

$$
\text { w.h.p. } \sum \varphi(A) \sim n^{3} p^{3} /(6 D) \sim n^{2} p / 6 \sim m / 3 .
$$

Finally, for $x, y \in V$, Theorem 2.4 bounds the probability that $x y$ (is in $G$ and) lies in at least $(1+\gamma) d$ triangles by $p \exp \left[-\gamma^{2} d /[2(1+\gamma / 3)]\right.$; so the expected number of triangles containing heavy edges is less than

$$
\left(\begin{array}{l}
n \\
2
\end{array}\right) p \sum_{i \geq 0} \exp \left[-2^{2 i} \varsigma^{2} d /\left(2\left(1+2^{i} \varsigma / 3\right)\right)\right]\left(1+2^{i+1} \varsigma\right) d=o\left(n^{3} p^{3}\right),
$$


and Markov's Inequality then gives (20).

Acknowledgment. We thank David Galvin for telling us the problem.

\section{REFERENCES}

[1] N. Alon and J. Spencer, The Probabilistic Method, 4th ed., Wiley Series in Discrete Mathematics and Optimization, John Wiley and Sons, Inc., 2016. 5, 11

[2] P. Bennett, R. Cushman, and A. Dudek, Closing the random graph gap in Tuza's Conjecture through the online triangle packing process, preprint. arXiv:2007.04478 [math.CO] 2

[3] P. Bennett, A. Dudek, and S. Zerbib, Large triangle packings and Tuza's conjecture in sparse random graphs, Combinatorics, Probability and Computing, to appear. 1, 2, 11

[4] P. Frankl and V. Rödl, Near perfect coverings in graphs and hypergraphs, Eur. J. Comb. 6 (1985), 317-326. 11

[5] P. Frankl and V. Rödl, Large triangle-free subgraphs in graphs without $K_{4}$, Graphs and Comb. 2 (1986), 135-144. 2

[6] Z. Füredi, Matchings and covers in hypergraphs, Graphs and Comb. 4 (1988), 115-206. 11

[7] D. Galvin, personal communication. 1

[8] P. Haxell, Packing and covering triangles in graphs, Discrete Math. 195 (1999), 251-254. 1

[9] P. Haxell, A. Kostochka and S. Thomassé, A stability theorem on fractional covering of triangles by edges, European J. Comb. 33 (2012), 799-806. 1

[10] S. Janson, T. Łuczak and A. Ruciński, Random Graphs, Wiley, New York, 2000. 2,5

[11] J. Kahn, A linear programming perspective on the Frankl-Rödl-Pippenger theorem, Random Structures $\mathcal{E}$ Algorithms 8 (1996), 149-157. 11

[12] R. Lyons and Y. Peres, Probability on Trees and Networks, Cambridge Series in Statistical and Probabilistic Mathematics, 42. Combridge University Press, New York, 2016. 4

[13] C. McDiarmid, On the method of bounded differences, Surveys in Combinatorics (1989), 148-188, London Math. Soc. Lecture Note Ser., 141, Cambridge Univ. Press, Cambridge, 1989. 5

[14] E.R. Scheinerman and D.H. Ullman, Fractional Graph Theory, Wiley, New York, 1997. 11

[15] J. Spencer, Asymptotic packing via a branching process, Random Structures and Algorithms 7 (1995), 167-172. 8, 9

[16] Zs. Tuza, Conjecture, Finite and Infinite Sets, Eger, Hungary 1981. A. Hajnal, L. Lovász, V.T. Sós (eds.) Proc. Colloq. Math. Soc. J. Bolyai, vol. 37, pp. 888. North-Holland, Amsterdam (1984). 1

[17] W. Vervaat, Upper bounds for the distance in total variation between the binomial or negative binomial and the Poisson distribution, Statistica Neerlandica 23 (1969), 79-86. 5

\section{Appendix A. Proof of Lemma 1.6}

There is nothing very interesting here and we aim to be brief. The lemma is easy when $d \geq 8$, since then $\xi(d)>1 / 4$ (while $\psi(d)<1 / 2$ for all $d$ ). For $d \in[1 / 2,8]$, we show the inequality in the form

$$
4(2 d+1)^{-1 / 2}-3 \exp \left(-\frac{d}{2}\left(1+e^{-d}\right)\right)<1 .
$$

Let

$$
f(d)=4(2 d+1)^{-1 / 2} ; \quad g(d)=3 e^{-d / 2} ; \quad h(d)=\exp \left(-\frac{d}{2} e^{-d}\right) .
$$

(So the 1.h.s. of (21) is $f-g h$.) It is easy to see that

$$
h(d) \text { is decreasing on }[0,1] \text { and increasing on }[1, \infty)
$$

and

$$
f \text { and } g \text { are convex. }
$$

Case 1: $d \in[k, k+1](k=1,2, \ldots, 7)$ 
Fix $k$ and let $f_{1}(\cdot)$ be the line through $(k, f(k))$ and $(k+1, f(k+1))$, and $g_{1}(\cdot)$ the tangent to $g$ at $(k+$ $1, g(k+1))$. From (22) and (23) we have

$$
f(d)-g(d) h(d) \leq f_{1}(d)-g_{1}(d) h(k),
$$

so it suffices to show (for $d \in[k, k+1]$ )

$$
f_{1}(d)-g_{1}(d) h(k)<1 .
$$

But the 1.h.s. of (24) is a linear function of $d$, so it's enough to check the inequality at the endpoints-which we won't, but for example, when $k=1$, the function is $A d+B$, with

(so (24) holds). Other $k^{\prime}$ s are similar.

$$
\begin{gathered}
A=\frac{4}{5} \sqrt{5}-\frac{4}{3} \sqrt{3}+\frac{3}{2} e^{-(1 / 2 e+1)}(\approx-0.06144)<0, \\
B=\frac{8}{3} \sqrt{3}-\frac{4}{5} \sqrt{5}-6 e^{-(1 / 2 e-1)}(\approx 0.99352)<1
\end{gathered}
$$

Case 2: $d \in[1 / 2,1]$

Here we take $f_{1}(\cdot)$ to be the line through $(1 / 2, f(1 / 2))$ and $(1, f(1))$, and $g_{1}(d)$ the tangent to $g$ at $(1, g(1))$, and again just need to show the analogue of (24), i.e.

$$
f_{1}(d)-g_{1}(d) h(1)<1 .
$$

The 1.h.s. of this is $A d+B$, with

$$
\begin{gathered}
A=\frac{8}{3} \sqrt{3}-4 \sqrt{2}+\frac{3}{2} e^{-1 / 2(1 / e+1)}(\approx-0.28111)<0, \\
B=4 \sqrt{2}-\frac{4}{3} \sqrt{3}-\frac{9}{2} e^{-1 / 2(1 / e+1)}(\approx 1.07664),
\end{gathered}
$$

so is maximized (on $[1 / 2,1]$ ) at $d=1 / 2$, where it is strictly less than 1 .

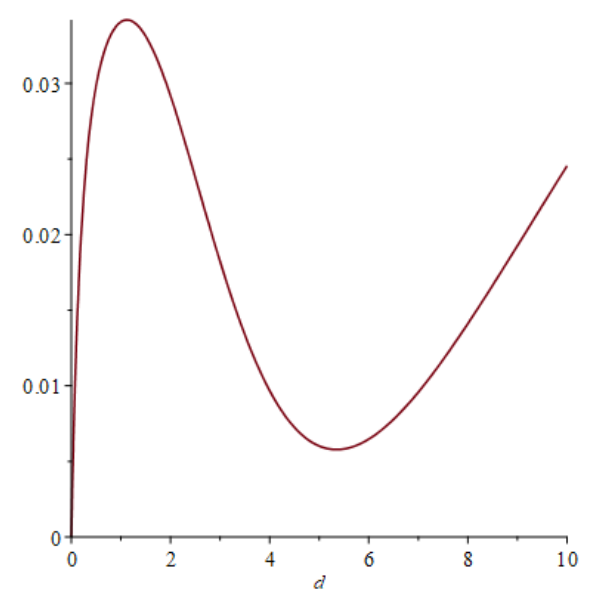

FIGURE 1. $2 \xi(d)-\psi(d)$ for $d \in[0,10]$

E-mail address: jkahn@math.rutgers.edu, jp1324@math.rutgers.edu

Department of Mathematics, Rutgers University, Hill Center for the Mathematical Sciences, 110 FrelinghuYSEN RD., PISCATAWAY, NJ 08854-8019, USA 